\title{
Covid-19 Rebooting medical education with virtual grand rounds during the COVID-19 pandemic
}

\author{
Authors: Dominic Sparkes, ${ }^{A *}$ Clare Leong, ${ }^{\mathrm{B} *}$ Katherine Sharrocks, ${ }^{C}$ Michael Wilson, ${ }^{\mathrm{D}}$ Elinor Moore ${ }^{\mathrm{E}}$ and \\ Nicholas ] Matheson ${ }^{F}$
}

Social distancing during the coronavirus disease 2019 (COVID-19) pandemic has necessitated drastic changes in the practice of hospital medicine, including the cancellation of many educational activities. At the same time, the emergence of a new disease with a rapidly evolving knowledge base has mandated timely educational updates. To resolve this conflict in our hospital, we substituted 'traditional' grand rounds with 'virtual' grand rounds delivered over Zoom, consisting of a local situation report and operational update, followed by a specialty-specific clinical presentation on management of COVID-19. Attendance was greatly increased (mean 384 attendees) compared to traditional grand rounds (mean 44 attendees) and included a diverse audience of medical professionals. Feedback was overwhelmingly positive, with $>80 \%$ of responders stating that the sessions would or might inform their clinical practice. COVID-19 has therefore provided an opportunity to modernise grand rounds, and develop a new model matching the needs of medical education beyond the pandemic.

KEYWORDS: COVID-19, SARS-CoV-2, pandemics, medical education, teaching rounds, infectious disease medicine

DOI: $10.7861 /$ fhj.2020-0180

Authors: Aregistrar in infectious diseases and medical microbiology at Cambridge University Hospitals NHS Foundation Trust, Cambridge, UK; ${ }^{B}$ academic clinical fellow in infectious diseases and medical microbiology at Cambridge University Hospitals NHS Foundation Trust, Cambridge, Uk; ' Cacademic clinical fellow in infectious diseases and general internal medicine at Cambridge University Hospitals NHS Foundation Trust, Cambridge, UK; ' ${ }^{D}$ clinical fellow (higher) in medical virology at Cambridge University Hospitals NHS Foundation Trust, Cambridge, UK; ${ }^{\mathrm{E}}$ Consultant in infectious diseases and general internal medicine at Cambridge University NHS Hospitals Foundation Trust, Cambridge, UK; F principal investigator at Cambridge Institute of Therapeutic Immunology and Infectious Disease (CITIID), University of Cambridge, UK, honorary consultant in infectious diseases at Cambridge University NHS Hospitals Foundation Trust, Cambridge, UK and honorary consultant in virology at NHS Blood and Transplant, Cambridge, UK. *These authors contributed equally

\section{Introduction}

The COVID-19 pandemic has necessitated drastic changes in social behaviour, in hospitals as well as public and private life. Attempts to reduce transmission have focussed on limiting unnecessary face-to-face contact between individuals, with far-reaching consequences for society., In healthcare settings, many services and processes have been restructured to focus on care for those affected. Activities seen as non-essential, especially those involving groups of people in enclosed spaces, have been greatly reduced or stopped altogether. Education in healthcare settings has been particularly affected, with widespread cancellations ranging from departmental meetings to international conferences. ${ }^{3,4}$

At the same time, the emergence of a new pandemic disease, with continually evolving clinical practice underpinned by a rapidly growing body of evidence, is exactly the sort of situation where effective and timely education for healthcare professionals is essential. This requirement is magnified further if clinicians are deployed outside their usual areas of expertise. Combined with the necessary reduction in educational events, there has therefore been a significant gap between what clinicians need, and what can be delivered. This has led to an increase in the use of virtual technology for education. ${ }^{2}$

'Traditional' (face-to-face) grand rounds have been a stalwart of medical education for more than a century, allowing clinicians to learn from interesting cases presented by colleagues. Their benefit has been extensively demonstrated, and they remain a vital tool for imparting medical knowledge. ${ }^{5,6}$ In this study, we therefore describe and evaluate our experience developing and delivering 'virtual' grand rounds at a large tertiary hospital in Cambridge, UK. This programme, comprising 15 weekly educational sessions themed around COVID-19 and broadcast to an online audience, filled the educational gulf resulting from the cessation of traditional grand rounds (and other educational activities) early in the pandemic.

\section{Methods}

Having obtained approval from the Grand Round Committee, we set out to provide an online COVID-19-themed educational programme at Cambridge University Hospitals NHS Foundation Trust (CUHNFT) on behalf of the infectious diseases team. We aimed to reach a diverse audience and offer timely updates for clinicians on both clinical practice and local and national policy relevant to COVID-19. 
Based on our previous experience using various digital platforms early in the pandemic, we selected Zoom (https://zoom.us/) as an option offering both a reliable service and easy access for the audience. Meeting details (including a link to join the meeting directly) were emailed by the grand rounds team to their usual mailing list, and additionally circulated to CUHNFT staff, medical students and local GPs.

Meeting settings were optimised to ensure minimum disruption, with participants prohibited from sharing their screen or using their own microphone, and the chat function limited to direct messages to the host. All presenters were instructed to remove patient-identifiable information from their presentations, and 'password' and 'waiting room' functions were used to avoid uninvited attendees. To further ensure smooth running of the technology, one member of the virtual grand round team was responsible for administrating the session via Zoom each week.

Sessions followed a traditional grand round format, with a chairperson introducing speakers and hosting a question and answer session at the end. Questions from attendees were posted directly to the chairperson using the chat function. Each weekly broadcast included a five-minute 'local update' from a member of the infectious diseases team, presenting the latest local and national COVID-19 case numbers and any relevant new evidence or operational policies, followed by a 25 -minute 'specialty presentation' from a specialist clinical team, discussing issues relevant to COVID-19 in their field. The programme covered a wide range of specialities, including neurology, haematology, immunology, cardiology, histopathology and emergency medicine. Each virtual grand round was recorded and uploaded to the CUHNFT staff website for further viewing.

Attendance numbers for virtual grand rounds were captured via Zoom (live viewings) and Vimeo (www.vimeo.com) (web viewings). Attendance numbers for traditional grand rounds from preceding years (recorded via swipe-card or paper registration on entry) were used as a comparator. Feedback was collected using an online questionnaire with a link to an online form (Google Forms) available at the end of each session. The feedback form included questions on basic demographics, specific feedback about the presentations (including whether they would inform the learner's clinical practice), technological issues, and the likelihood of attendance at future sessions. Additional comments and feedback were received via email. Data were collated and analysed using Microsoft Excel and displayed using GraphPad Prism.

\section{Results}

Virtual grand rounds commenced on 22 April 2020 and ran weekly thereafter for 15 sessions. The mean weekly number of live viewers was 384 (range 198-1,025), compared to a mean of 44 viewers per week (range 1-75) for grand rounds held during the same period from 2015-2019 (Fig 1). There was an exceptionally high attendance initially $(n=1,025)$, which declined for 4 weeks before plateauing from week 5 (range 198-369). Because virtual grand rounds were recorded and uploaded to the CUHNFT website, additional web viewings were possible, and contributed significantly to the overall attendance.

As of 3 Aug 2020, 188 feedback forms had been completed. Some forms contained feedback for more than one session, giving a total of 280 responses relating to individual sessions. The majority of respondents were hospital physicians, but GPs and other health professionals were also well-represented (consultants $n=70$, junior doctors $n=45$, GPs $n=28$, medical students $n=18$ and other professionals $n=27$ ). $90.5 \%$ of respondents reported their location as either Cambridge or the East of England, with the remainder based elsewhere in the UK or Europe (with one respondent located in Indonesia).

Feedback was overwhelmingly positive, indicating that the local updates were 'very informative' (72.7\% of responses) and 'understood fully' ( $69.3 \%$ of responses). Similar figures were obtained for the specialty presentations, indicating that they were 'very informative' (69.2\% of responses) and 'understood fully' ( $52.3 \%$ of responses). $84.8 \%$ of the responses were 'yes' or 'maybe' when asked if the local updates would inform their clinical practice and $83.1 \%$ of responses the same for the specialty presentations (Fig 2).

Feedback regarding the technology indicated that it posed very few problems to users: $92.6 \%$ of respondents reported no difficulty accessing the talk, with $94.8 \%$ confirming that the slides were

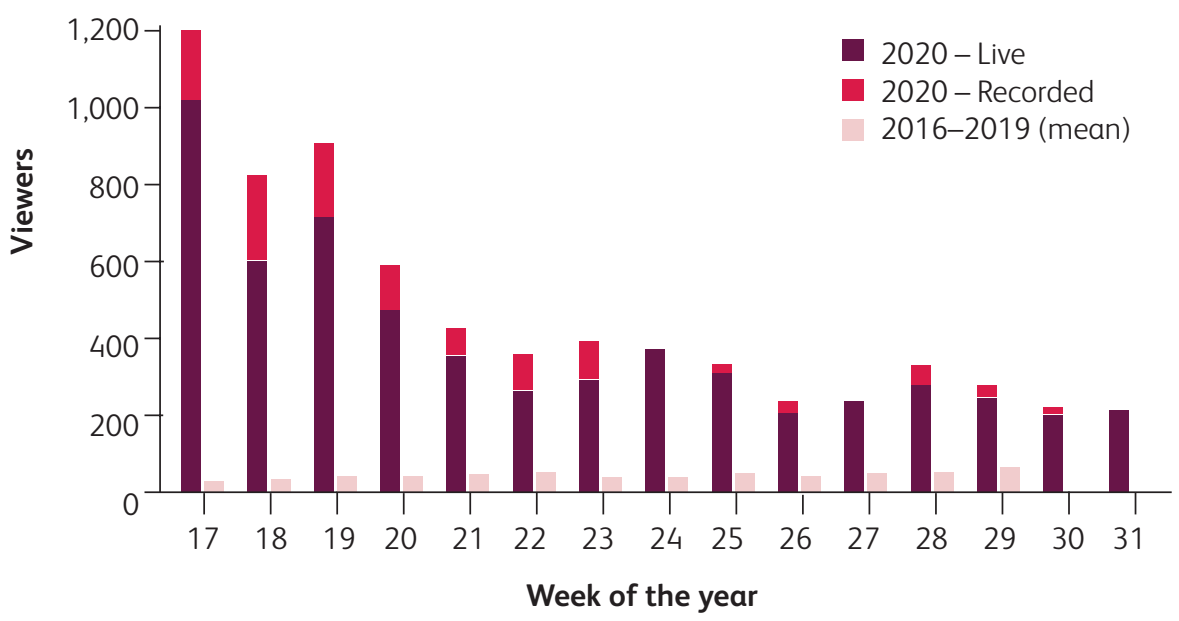

Fig 1. Total recorded attendances at grand rounds by week of the year. Red stacked bars show the total attendance in 2020 (dark red = live viewers, light red = website views). Pink bars show the mean attendance by week of the year from 2016-2019. No web views occurred in weeks 24 and 27 due to a lack of recording. No grand rounds took place in 2016-19 on weeks 30 or 31. 
a

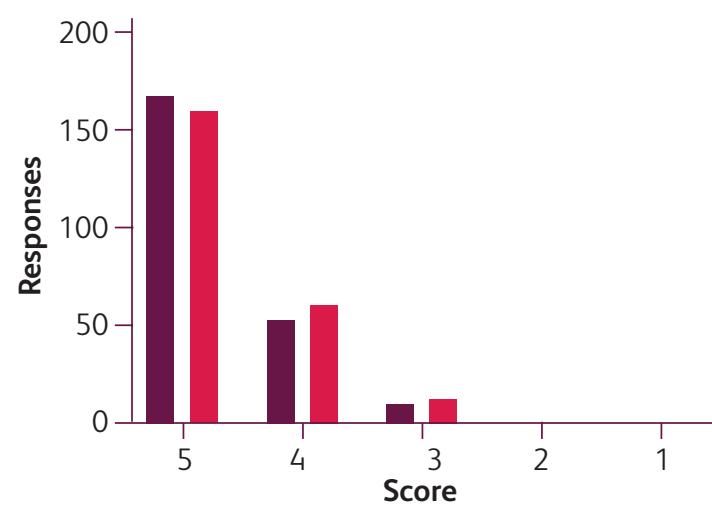

b

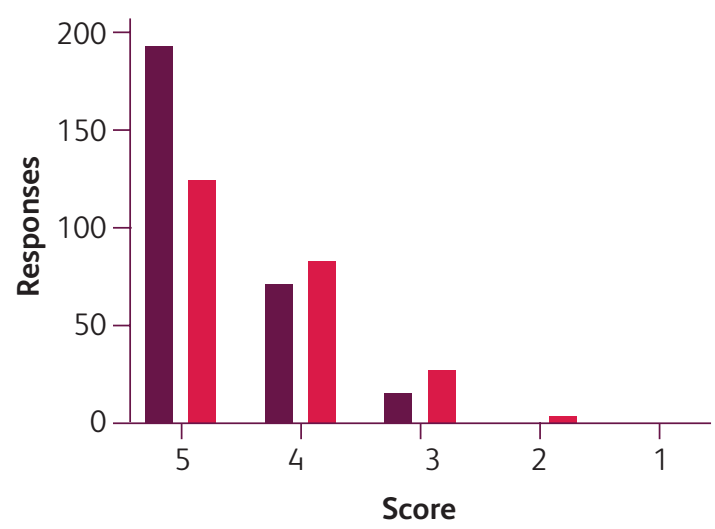

To what extent did you find the specialty presentation informative?

To what extent did you understand the information in the specialty presentation?

Fig 2. Cumulative feedback using a score of $1-5$ to assess how informative and understandable (a) the local updates and (b) the specialty presentations were.

transmitted adequately and $97.8 \%$ that the presenter's voice was audible. $88.9 \%$ of respondents stated that they would be very likely to attend a future virtual grand round.

Free text feedback on the questionnaire and received via email was extremely positive, expressing praise both for individual speakers and the overall programme, appreciation for the work involved in delivering the programme, and multiple requests for 'virtual' attendance at grand rounds to continue to be available in future. Some examples are highlighted in Box 1.

\section{Discussion}

It is a central tenet of medical professionalism that doctors keep their knowledge and skills up to date.? This becomes even more important in the context of a pandemic of a previously unknown disease. Bridging the gap between the (increased) need for education and the (decreased) provision of education during the COVID-19 pandemic, the virtual grand rounds described in this study provided a new educational programme which could be

\section{Box 1. Representative written comments from} feedback forms and emails, grouped by theme

The ability to access content online:

'It is so much easier to 'attend' a grand round via Zoom pandemic aside. It would be great to continue them online postCOVID. It allows those either not based in Cambridge or without time to attend in person to benefit from them. I've not been to so many grand rounds in years as I have done in the past few months. Well done to everyone involved.'

'Excellent organisation and great bite size value; not usually able to attend grand rounds... but this has a far greater reach and I hope is continued beyond these exceptional times when we are back to normal service.'

\section{Feedback from GPs:}

'Please, please continue offering this for local GPs - even the grand rounds when COVID is over will be educational and the ability to ask questions and bridge the primary/ secondary care interface is very much appreciated. Outstanding series so far - has been very helpful in getting a handle on COVID and the impact on the system locally.'

'I am a GP, hugely value being able to join the grand round. Please keep this going particularly beyond COVID-19. Thank you.'

\section{General comments:}

'Excellent as usual. Concise and informative, these seminars have been consistently very interesting.'

'Overall I've found these succinct and super-informative week by week.'

widely accessed, without breaching social distancing measures. The exceptionally high attendance at the first few sessions may have reflected a combination of factors: the fact that they coincided with large numbers of cases being admitted locally and nationally, a dearth of other educational opportunities at the time, and (possibly) a degree of 'lockdown fatigue', causing people to seek novel opportunities. Attendance declined over the first few weeks of the programme, likely because of a reduction both in the need for education and interest in the topic, as clinicians became more comfortable caring for COVID-19 patients, and case numbers declined. Even so, it remained much higher than at traditional grand rounds from preceding years.

One limitation of our data is that the recorded attendance numbers only represent estimates of 'true' attendance. The Zoom report collects data on individual 'entries' to the meeting, so individuals leaving and re-joining the meeting may be counted twice; these numbers may therefore represent an over-estimate of 'true' attendance. Likewise, attendance data for grand rounds in preceding years, captured by swipe-card or paper registration, exclude attendees who fail to register; these numbers may therefore represent an under-estimate of 'true' attendance.

Nonetheless, the magnitude of the difference between recorded attendances at virtual and traditional grand rounds strongly suggest that the virtual grand rounds attracted much larger audiences overall. In addition, whilst the response rate was modest, feedback indicated that respondents overall had very positive reactions to the programme (level 1 in Kirkpatrick's evaluation mode ${ }^{8}$ ), and that it would inform their clinical practice (Kirkpatrick's 
level 3). Taken together, the combination of high recorded attendances, and positive feedback from attendees, provides strong evidence that attendees found the programme to be beneficial. Furthermore, multiple comments received in feedback about the value of remote access to grand rounds indicate that the opportunity to attend virtual grand rounds online has intrinsic benefit, rather than simply functioning as an imperfect alternative when traditional grand rounds are not possible.

Virtual grand rounds and conferences have been trialled previously. ${ }^{9}$ The use of video lectures with interactive questions has also been demonstrated using other modalities, including Twitter, allowing people from around the country and world to participate and learn, uninhibited by their location. Our attendance data suggest that virtual grand rounds may facilitate access to a wider range of medical professionals than is possible with in-person attendance. While acknowledging that demographic data were not collected over previous years, it is unlikely that communitybased healthcare professionals (including GPs) would have been able to attend regularly prior to this.

Traditional grand rounds are known to have specific benefits. Direct visual feedback from an audience can be valuable to the presenter, and help to improve presentation delivery. They also represent an opportunity for networking, and for direct clinical discussion between specialties before and after sessions. We therefore propose a method of implementing grand rounds in the future which combines both formats, in which a speaker delivers a presentation in a lecture theatre with an audience, live-streamed to virtual attendees (and recorded, to allow for online viewing at a later date). We intend to implement this 'live-plusvirtual' model at our Trust when social distancing restrictions are no longer required. This amalgamation of grand round formats will allow it to match the needs of all medical professionals, for COVID-19 and beyond.

\section{References}

1 Government Guidance. Staying alert and safe (social distancing). Cabinet Office, 2020. www.gov.uk/government/publications/ staying-alert-and-safe-social-distancing/staying-alert-and-safesocial-distancing-after-4-july [Accessed 30 August 2020].

2 Jarvis CI, Van Zandvoort K, Gimma A et al. Quantifying the impact of physical distance measures on the transmission of COVID-19 in the UK. BMC Med 2020;18:124.

3 Gill D, Whitehead C, Wondimagegn D. Challenges to medical education at a time of physical distancing. Lancet 2020;396:77-9.

4 Kachra R, Brown A. The new normal: medical education during and beyond the COVID-19 pandemic. Can Med Ed J, in press (doi: 10.36834/cmej.70317).

5 Hull AL, Cullen RJ, Hekelman FP. A retrospective analysis of grand rounds in continuing medical education. J Contin Educ Health Prof 1989;9:257-66.

6 Stites SD, Warholic CL. Multicultural grand rounds: competencybased training model for clinical psychology graduate students. Psychol Learning Teaching 2014;13:261-9.

7 General Medical Council. The duties of a doctor registered with the General Medical Council. GMC, 2020. www.gmc-uk.org/ ethical-guidance/ethical-guidance-for-doctors/good-medical-practice/duties-of-a-doctor [Accessed 30 August 2020].

8 Kirkpatrick, DL. Evaluating training programs: the four levels. San Francisco: Berrett-Koehler, 1994

9 Laochamroonvorapongse D, Johnson E, Foong HB, Elpern DJ. A brave new world: virtual grand rounds in dermatology. Semin Cutan Med Surg 2002;21:232-6

Address for correspondence: Dr Dominic Sparkes, Clinical Microbiology and Public Health Laboratory, Level 6, Addenbrooke's Hospital, Hills Road, Cambridge CB2 0QW, UK. Email: dominic.sparkes@addenbrookes.nhs.uk 